\title{
MALDI-TOF/TOF mass spectrometry for determination of yeast diversity in traditional cornelian cherry tarhana produced with different cereal/pseudocereal flours
}

\author{
Asiye Elvan Bellici ${ }^{1} \cdot$ Seda Karasu-Yalcin ${ }^{1} \cdot$ Kubra Eryasar-Orer $^{1} \cdot$ Erkan Yalcin $^{1}$
}

Received: 12 October 2018 / Accepted: 18 February 2019/Published online: 1 March 2019

(C) Università degli studi di Milano 2019

\begin{abstract}
Purpose This study is to determine the dynamics in the microflora of cornelian cherry tarhana (CCT), a traditional food of Turkey, by MALDI-TOF/TOF MS.

Methods Non-fermented and fermented CCT productions were performed by using flours of bread wheat, wholegrain hull-less barley, buckwheat, and clear flour. Identification of the isolates obtained from raw materials and various production steps of the CCT was performed by MALDI-TOF/TOF MS. Dendograms were prepared by cluster analysis and the distances between the strains isolated during production stages and from cornelian cherry puree were determined.

Results Totally, 231 isolates were obtained and $45.5 \%$ of them were identified at species level, $30.3 \%$ at genus level while $24.2 \%$ of the isolates could not be identified. It was found that microflora of cornelian cherry tarhana is mainly composed of yeasts. Thirty-two percent of the identified yeast isolates were Hanseniaspora uvarum and the others were Saccharomyces cerevisiae (19.6\%), Torulaspora delbrueckii (18.6\%), Candida krusei (11.3\%), Candida lusitaniae (9.3\%), Metschnikowia pulcherrima (3.1\%), Wickerhamomyces anomalus (2.1\%), Candida kefyr (2.1\%), Cyberlindnera fabianii (1\%), and Candida parapsilosis (1\%). Only two lactic acid bacteria could be isolated, which were Lactobacillus reuteri and Enterococcus spp., originating from buckwheat flour and clear flour, respectively. Dendograms revealed that some of the yeast strains isolated during production were originating from cornelian cherry.

Conclusions Microflora of CCT was investigated for the first time. This is one of the few studies using MALDI-TOF/TOF MS for identification of food originated yeasts. Novel species-identified, endogenic yeasts which could have potential technological characteristics were introduced.
\end{abstract}

Keywords Cornelian cherry tarhana $\cdot$ Yeast $\cdot$ Lactic acid bacteria $\cdot$ MALDI-TOF/TOF MS

\section{Introduction}

Fermented foods and beverages were among the first processed food products consumed by humans. Fermentations could be described based on microorganisms involved, such as yeast, lactic acid bacteria (LAB), and acetic acid bacteria, and based on food substrates, which include meats and fish, dairy, fruits and vegetables, legumes, and cereals. Due to the rich variety of food-microorganism combinations, there are

Seda Karasu-Yalcin

yalcin_s@ibu.edu.tr

1 Faculty of Engineering, Department of Food Engineering, Bolu Abant Izzet Baysal University, Golkoy Campus, 14030 Bolu, Turkey thousands of national or international fermented foods and beverages (Marco et al. 2017). Among the cereal-based fermented foods, bread and beer are the most popular ones. But, there are also many other products prepared in different regions of the world (tempeh, pito, sekete, kwass and etc.) and in Turkey (boza, tarhana, kumru, chickpea bread, and etc.). Most of these products are manufactured in homes, villages, and/or small-scale industries by using either wheat, maize, barley, oat, millet, rice, or their flours (Tanguler et al. 2014).

Tarhana is an old and popular traditional Turkish fermented product which is generally consumed as soup. It is produced mainly by mixing wheat flour, yoghurt, yeast, and a variety of cooked vegetables and spices (tomato, onion, salt, mint, paprika), followed by fermentation, drying, and grinding (Ozdemir et al. 2007; Tanguler et al. 2014). According to the Turkish Standardization Institute, there are four categories of 
tarhana based on the production method employed, which are "flour tarhana," "goce tarhana", "semolina tarhana," and "mixed tarhana." The use of wheat flour, chopped wheat, and semolina separately or as combinations in the recipe makes them different (Kabak and Dobson 2011). However, there are also various studies which other cereal and legume flours such as barley, buckwheat, corn, lupin, and rice are used in the production of tarhana. On the other hand, some plant and fruits such as cherry laurel, bilberry fruit, grape, and cornelian cherry could be added to formulation of traditional wheat flour tarhana (Cagindi et al. 2016).

Cornelian cherry tarhana, also named as sour tarhana, is traditionally produced in a few number of provinces including Bolu, and also having a geographical mark in Bolu. The major distinction of the CCT is that yoghurt is not used in the tarhana formulation. Additionally, the CCT productions are usually performed without fermentation, except one type of the CCT produced in Kutahya province. Briefly, the tarhana dough is prepared by mixing of wheat flour and raw or cooked cornelian cherry or its puree at a certain ratio. Kneading is applied, followed by drying. There are only a few studies about CCT, because it is domestic and produced in a limited area. On the other hand, so many studies could be found in the literature about the phytochemicals and their chemical structures and also pharmacological activities of the cornelian cherry (Dinda et al. 2016).

Every traditional fermented food has a specific microflora and fermented foods serve as a good source of probiotic and functional microorganisms which could have important physiological properties. Tarhana fermentation is accomplished with LAB and yeasts and there are several reported studies about determination of tarhana microflora (Sengun et al. 2009; Settanni et al. 2011; Ozel et al. 2015; Simsek et al. 2017). However, there is no reported study about the microflora of CCT. In this study, CCT was produced traditionally and also by applying fermentation. Productions were performed by using flours of bread wheat, wholegrain hull-less barley, buckwheat, and clear flour. Fermentation was applied for the first time and it was aimed to determine the effects of fermentation as well as using different raw materials on the microflora. Matrix-assisted laser desorption ionization time-of-flight/ time-of-flight mass spectrometry (MALDI-TOF/TOF MS) was used for microbial identifications. Similarity levels between the isolates of the same species were determined by using the dendrograms.

\section{Materials and methods}

\section{Raw materials}

For the production of cornelian cherry tarhana (CCT), flours of bread wheat, hull-less barley, buckwheat, and clear flour (a by-product of semolina industry), uncooked cornelian cherry puree, and salt were used as ingredients. Hull-less barley (Ozen cv.) was provided from the Central Research Institute of Field Crops (Ankara, Turkey) and grinded to wholegrain hull-less barley flour by using stone mill (A700 Genuine Wood, Good Mills Company, Osttirol, Lienz, Austria). Buckwheat (Gunes $c v$.) was obtained from the Bahri Dagdas International Agricultural Research Institute (Konya, Turkey) and it was grinded to buckwheat flour by using laboratory roller-mill (Quadrumat Junior, Brabender GmbH \& Co., Duisburg, Germany). Clear flour was supplied from the semolina milling unit of a local pasta company. The cornelian cherry (Cornus mas L.) was obtained from local producers in the Bolu province. Cornelian cherry puree was prepared in the production plant of local company in Bolu.

\section{Cornelian cherry tarhana production}

Productions of CCT by using bread wheat (traditional), wholegrain hull-less barley, and buckwheat flours and clear flour were performed in one of the company's production plant. CCT samples were produced also by applying fermentation, besides using traditional production procedure without fermentation. The formulation of the cornelian cherry tarhana doughs and production steps were determined according to the proposals of the CCT producers. Traditional CCT was produced by using bread wheat flour [puree $(52.7 \%)$, flour (42\%), salt (5.3\%)], wholegrain hull-less barley flour [puree (59.4\%), flour (34.7\%), salt (5.9\%)], buckwheat flour [puree $(55.3 \%)$, flour $(39.2 \%)$, salt $(5.5 \%)$, and clear flour [puree $(58 \%)$, flour (36.2\%), salt (5.8\%)]. For each of the formulation, the ingredients were mixed in the specified quantities and homogenously kneaded (KitchenAid, USA) to make tarhana doughs in similar dough consistencies.

The tarhana dough samples were spread on the separate clothes in the portions of approximately $20 \mathrm{~g}$ and dried at $25{ }^{\circ} \mathrm{C}$ and $60 \%$ humidity for 2 days. The dried products were grinded and they were left to final drying for 6 days under the same conditions. At the end of this period, the CCT samples produced from the different kinds of flours were again grinded to obtain the final non-fermented products.

For preparing the fermented tarhana samples, fermentation process was applied to the kneaded tarhana doughs in covered boxes at $25{ }^{\circ} \mathrm{C}$ for 3 days. Fermentation step was performed without adding any starter culture. After the fermentation step, drying, grinding, and the final drying procedures were applied at the same conditions as used for the non-fermented products. The non-fermented and fermented products were taken into polyethylene bags and they were stored in the refrigerator conditions until analysis. 


\section{Sampling procedure}

Samples from raw materials and different production steps were taken to investigate the changes in the yeast and lactic acid bacteria (LAB) microflora of the non-fermented and fermented CCT products. The samples including tarhana dough, dried product, and final product were taken from the non-fermented tarhana. Additionally, samples at the 24th, 48th, and 72nd hours of the fermentation process were taken from the fermented tarhana. After the sampling, the isolation and identification of the microorganisms were carried out.

\section{Isolation of yeast and LAB}

Twenty-five grams of samples were homogenized in $225 \mathrm{~mL}$ of $0.1 \%$ sterile peptone water, then serial dilutions were prepared. For yeast enumeration and isolation, each dilution was spread on YGC (yeast extract glucose chloramphenicol) agar plates composed of $(\mathrm{g} / \mathrm{L})$ : yeast extract, 5; glucose, 20; chloramphenicol, 0.1; and agar, 14.9. The number of yeasts was determined and the different colony morphologies were selected from countable plates, following the incubation at $28^{\circ} \mathrm{C}$ for $48 \mathrm{~h}$. Cultures were activated in YM (yeast extract malt extract) broth and microscopic morphologies of the yeasts were examined. The colonies were purified by restreaking on the YM (yeast extract malt extract) agar. Cultures were stored at $4{ }^{\circ} \mathrm{C}$ in $\mathrm{YM}$ agar and activated in the same medium.

LAB isolation was performed by using MRS and M17 agar. The plates for MRS agar were incubated under microaerophilic conditions and the M17 agar plates were incubated aerobically at $37{ }^{\circ} \mathrm{C}$ for $24-48 \mathrm{~h}$. The colony morphologies were observed and the different ones were purified on their isolation medium (MRS or M17) agar. Purified cultures were stored in $20 \%$ glycerol at $-20{ }^{\circ} \mathrm{C}$ and the stock cultures were cultivated for further experiments. Additionally, Gram staining and catalase test were applied to the isolates from MRS and M17 agar. At this stage, Gram-positive and catalase-positive cultures were selected and passed on the subsequent step.

\section{Identification by MALDI-TOF/TOF MS}

In this study, identifications of the isolated lactic acid bacteria and yeast cultures were performed by using MALDI-TOF/ TOF MS in Scientific Industrial and Technological Application and Research Center of Bolu Abant Izzet Baysal University, Turkey. Direct transfer method, direct transferformic acid method, and extraction method were used for the identification of the microorganisms with the Bruker MALDI-TOF/TOF MS (Autoflex Speed Bruker Daltonics, Germany). The direct transfer method was described by Schulthess et al. (2013) as transferring of fresh colony onto target plate's spot and being overlaid with 4-hydroxy- $\alpha$ cyanocinnamic acid ("alphacyano" or 4-HCCA) matrix. However, majority of the isolates could not be identified with this method; direct transfer-formic acid method was carried out, which $70 \%$ formic acid was added to spots before the matrix solution. The considerable majority of the microorganisms were identified by using this given method. To identify the unknown cultures, another proposed extraction procedure was followed. In this method, a fresh colony was suspended in deionized water and ethanol was added; and after centrifugation of the cell suspension, the supernatant was discarded and the pellet was resuspended in $70 \%$ formic acid and acetonitrile. Following the second centrifugation, the supernatant was transferred to the spot before being overlaid with 4-HCCA matrix (Schulthess et al. 2013).

Mass spectra were processed using BioTyper software (version 3.1; Bruker Daltonics). As reported by Nacef et al. (2017), matching between experimental profiles obtained from the microorganism isolates and the reference profiles is expressed by BioTyper according to a score. It was briefly explained that if a score is higher than 2.0, it means identification at the species level and the value between 1.7 and 2.0 imply only genus identification. On the other hand, the score value under 1.7 shows no significant similarity between the unknown profile and the database (Nacef et al. 2017). Cluster analysis was performed for MALDI-TOF/TOF main mass spectra (MSP) of yeast strains originated from cornelian cherry puree and from different kinds of cornelian cherry tarhana. Dendograms of identified strains were generated with the Biotyper MSP Dendogram Creation Standard Method. The mass spectra of common yeast species (H. uvarum, C. lusitaniae, C. krusei, and S. cerevisiae) which were originated from cornelian cherry puree were chosen for the dendograms. Four different dendograms were prepared for each CCT production. Each of the dendogram indicated the distance level between cornelian cherry puree strains and the others from different stages of CCT production. It was aimed to screen a yeast strain obtained from any production stage.

\section{Results}

A total of 231 isolates were obtained at the production stages of the non-fermented and fermented CCT samples made from cornelian cherry and four different kinds of flours. As an outcome of the identification by using MALDI-TOF/TOF MS, 161 of the total 231 microorganisms were determined to be yeasts and species (97) and genus (64) identifications of them were carried out. Fourteen of the isolates were detected to be in different genera of bacteria including Bacillus (11), Kosakonia (1), Enterococcus (1), and Lactobacillus (1). However, identification of 56 isolates could not be possible, corresponding to $24.2 \%$ of total number. Overall analyzing 
results indicated that $45.5 \%$ and $30.3 \%$ of the isolates were identified at species and genus levels, respectively.

The yeast counts of the cornelian cherry puree, wholegrain hull-less barley, and buckwheat flours and clear flour were found as $6.36,4.30,4.61$, and $3.70 \log \mathrm{cfu} / \mathrm{g}$, respectively. However, yeast growth could not be observed in the wheat flour. The yeast counts of the four non-fermented tarhana doughs were found to change between 4.81 and $5.14 \mathrm{log}$ $\mathrm{cfu} / \mathrm{g}$ and these values were in the range of 3.00-3.54 log $\mathrm{cfu} / \mathrm{g}$ for the final products of CCT. On the other hand, it was clearly observed that the fermentation process leads to an increase in the yeast counts of the tarhana doughs. The tarhana doughs contained fewer amounts of yeasts (4.46-
$5.62 \log \mathrm{cfu} / \mathrm{g}$ ) at the beginning, compared to the doughs at the 72nd hours of the fermentation (6.24-6.38 log cfu/g). The yeast counts decreased after the drying stages of fermented CCT products, similarly to the non-fermented CCT (data not shown).

The obtained MALDI-TOF/TOF MS profiles of the isolates were compared to the reference spectra of the BioTyper database for their similarity, and then, the isolates were identified at species or genus levels regarding to the given BioTyper score values. Identification results obtained during CCT productions were given in Table 1. Eight isolates of cornelian cherry puree were identified as Hanseniaspora uvarum (4), Saccharomyces cerevisiae (1), Candida

Table 1 Identification results of the isolates obtained by MALDI-TOF/TOF MS

\begin{tabular}{|c|c|}
\hline Raw materials/products & Identified isolates \\
\hline Cornelian cherry puree & $\begin{array}{l}\text { H. uvarum MP-1, MP-2, MP-3, MP-5; S. cerevisiae LP-6; C. lusitaniae LP-7, LP-9; C. krusei LP-2; } \\
\text { *S. cerevisiae LP-8; *C. krusei LP-11 }\end{array}$ \\
\hline Buckwheat flour & L. reuteri LKBU-4; K. cowanii LKBU-3 \\
\hline Clear flour & C. fabianii MIAU-1; *C. fabianii LIAU-6; *Enterococcus faecium LIAU-2 \\
\hline $\begin{array}{l}\text { Non-fermented CCT produced with wheat } \\
\text { flour }\end{array}$ & $\begin{array}{l}\text { H. uvarum MKH-1, MKD-2; T. delbrueckii MKH-3, MKD-1; S. cerevisiae MKH-2, MKH-4, LKH-3; } \\
\text { M. pulcherrima MKD-3; C. krusei LKH-1; C. lusitaniae LKH-4; }{ }^{*} \text { C. lusitaniae LKH-6; }{ }^{*} \text {. krusei } \\
\text { LKD-1, LKD-5, LKD-6 }\end{array}$ \\
\hline Fermented CCT produced with wheat flour & $\begin{array}{l}\text { H. uvarum MFKHc-2, MFKD-2; T. delbrueckii MFKS-1, MFKS-2; M. pulcherrima MFKD-1; C. krusei } \\
\text { LFKH-1, LFKHc-1, LFKD-2; *C. lusitaniae MFKH-1, LFKH-6; *C. krusei LFKH-7; *M. } \\
\text { pulcherrima MFKH-3, MFKHa-1, MFKHb-3, MFKS-3;*S. cerevisiae LFKHc-6; *P. kluyveri } \\
\text { LFKH-5 }\end{array}$ \\
\hline $\begin{array}{l}\text { Non-fermented CCT produced with } \\
\text { wholegrain hull-less barley flour }\end{array}$ & $\begin{array}{l}\text { H. uvarum MAH-1; S. cerevisiae MAH-5, MAD-1, MAD-2, LAH-4, LAH-8; T. delbrueckii MAH-3, } \\
\text { MAD-3; C. lusitaniae LAH-6, LAD-1; *S. cerevisiae MAH-4, MAD-5; *M. pulcherrima MAD-4 }\end{array}$ \\
\hline $\begin{array}{l}\text { Fermented CCT produced with wholegrain } \\
\text { hull-less barley flour }\end{array}$ & $\begin{array}{l}\text { H. uvarum MFAH-2, MFAHa-1, MFAHb-3, MFAHc-2; T. delbrueckii MFAHa-2, MFAS-1; } \\
\text { M. pulcherrima MFAHc-1; } S \text {. cerevisiae LFAD-5; C. kefyr LFAH-6; *M. pulcherrima MFAH-1, } \\
\text { MFAHa-4, MFAHb-2, MFAD-2, MFAS- } 2 ; * \text {. lusitaniae LFAHb-6, LFAHb-1, LFAD-7, LFAS-2; } \\
\text { *C. krusei LFAHb-5, LFAD-6; *C. guilliermondii LFAHa-2; *P. kluyveri MFAHa-3, MFAHb-1; }{ }^{*} \text {. } \\
\text { cerevisiae LFAD-4 }\end{array}$ \\
\hline $\begin{array}{l}\text { Non-fermented CCT produced with } \\
\text { buckwheat flour }\end{array}$ & $\begin{array}{l}\text { H. uvarum MKBH-4, MKBD-2; S. cerevisiae MKBH-1, MKBD-4, LKBH-6; T. delbrueckii MKBH-5, } \\
\text { MKBD-1; *M. pulcherrima MKBD-3; }{ }^{*} \text { S. cerevisiae LKBD-9, LKBD-2, LKBD-7; B. thuringiensis } \\
\text { LKBH-4 }\end{array}$ \\
\hline $\begin{array}{l}\text { Fermented CCT produced with buckwheat } \\
\text { flour }\end{array}$ & $\begin{array}{l}\text { H. uvarum MFKBH-2, MFKBHa-2-1, MFKBHa-4, MFKBHb-3, MFKBHc-2; T. delbrueckii } \\
\text { MFKBH-1; W. anomalus MFKBHb-2; S. cerevisiae MFKBD-3, LFKBHc-1; C. krusei LFKBHa-8, } \\
\text { LFKBHc-8, LFKBD-3; C. lusitaniae LFKBHa-4; C. kefyr LFKBH-9; *C. krusei MFKBHa-1; *C. } \\
\text { lusitaniae LFKBH-6, LFKBH-8, LFKBHc-2; *C. valida MFKBHa-2-2; *M. pulcherrima } \\
\text { MFKBHa-3, MFKBHb-1, MFKBHc-1, MFKBD-2, MFKBD-4, MFKBS-1; *S. cerevisiae } \\
\text { LFKBHa-7, LFKBHb-10, LFKBD-1; *H. uvarum MFKBD-1; *Bacillus pumilus LFKBH-1; } \\
\text { *B. licheniformis LFKBS-2 }\end{array}$ \\
\hline $\begin{array}{l}\text { Non-fermented CCT produced with clear } \\
\text { flour }\end{array}$ & $\begin{array}{l}\text { H. uvarum MIAH-1, MIAH-3, MIAH-7, MIAD-1; S. cerevisiae MIAH-5, MIAH-6, MIAH-8, LIAH-1; } \\
\text { T. delbrueckii MIAH-2, MIAD-2, MIAS-1, MIAS-4; C. lusitaniae LIAH-10; *M. pulcherrima } \\
\text { MIAD-3; *C. lusitaniae LIAD-8; B. oleronius LIAH-6; *B. cereus LIAD-5 }\end{array}$ \\
\hline Fermented CCT produced with clear flour & $\begin{array}{l}\text { H. uvarum MFIAH-3, MFIAH-5, MFIAHa-1, MFIAHb-1, MFIAHc-1, MFIAHc-2, MFIAD-2; } \\
\text { T. delbrueckii MFIAHa-4, MFIAHb-2, MFIAS-3; C. krusei MFIAH-1, LFIAHb-9, LFIAH-4; } \\
\text { W. anomalus MFIAHb-4; C. parapsilosis LFIAHb-8; C. lusitaniae MFIAH-6, LFIAH-5; *C. valida } \\
\text { MFIAH-8; *C. lusitaniae LFIAHc-2, LFIAHc-5, LFIAD-4; *M. pulcherrima MFIAHa-2, } \\
\text { MFIAHb-3, MFIAS- } 2 ; * \text { W. anomalus MFIAD-1; * S. cerevisiae LFIAHb-4; B. cereus LFIAH-1, } \\
\text { LFIAHc-7; B. licheniformis LFIAH-3, LFIAD-6; } * \text { B. pumilus LFIAHa-1; *Bacillus circulans } \\
\text { LFIAS-1 }\end{array}$ \\
\hline
\end{tabular}

*Identified at genus level 
lusitaniae (2), and Candida krusei (1). Furthermore, two isolates were identified at genus level which were $S$. cerevisiae and $C$. krusei. As an example, mass spectra of the isolates $H$. uvarum MP-1 and S. cerevisiae LP-6 were given in Fig. 1. In addition, one isolate was identified as Lactobacillus reuteri and one isolate of Kosakonia cowanii was encountered in buckwheat flour. In clear flour, two yeast strains belonged to Cyberlindnera fabianii (at levels of 1 species and 1 genus) and one LAB was identified as Enterococcus spp. Furthermore, no isolates could be obtained from the flours of wheat and hull-less barley.

For the CCT with wheat flour, 10 isolates at species level (H. uvarum, 2; S. cerevisiae, 3; Torulaspora delbrueckii, 2; Metschnikowia pulcherrima, 1; C. krusei, 1 ; and $C$. lusitaniae, 1) and 4 Candida spp. were identified (Table 1). However, 6 of the total 20 isolates could not be identified (score $\leq 1.7)$. In fermented CCT with wheat flour, 17 of the total 31 isolates could be identified and based on the species-level identification (8) results, the isolates were H. uvarum (2), T. delbrueckii (2), M. pulcherrima (1), and C. krusei (3). In addition, 9 of the 17 isolates were identified at genus level and they were given as Candida (3), Metschnikowia (4), Pichia (1), and Saccharomyces (1).

With regard to the identification results of the isolates obtained from the non-fermented CCT with wholegrain hull-less barley flour, 13 of the total 16 yeast isolates were identified. Ten of the isolates belonged to species of H. uvarum (1), S. cerevisiae (5), T. delbrueckii (2), and C. lusitaniae (2), while the other three isolates were in the genera of Saccharomyces (2) and Metschnikowia (1). For the fermented CCT with wholegrain hull-less barley flour, 9 yeast isolates (H. uvarum, 4; T. delbrueckii, 2; M. pulcherrima, 1; S. cerevisiae, 1; Candida kefyr, 1) were identified at species level. Besides, genera of 15 isolates including Candida (7), Metschnikowia (5), Pichia (2), and Saccharomyces (1) were performed and seven of the total 31 isolates could not be identified (Table 1).

For the non-fermented CCT with buckwheat flour, species identifications of 8 of the 12 isolates were achieved. Seven of the identified strains were yeasts (H. uvarum, 2; S. cerevisiae, 3; and T. delbrueckii, 7) and one of them was bacteria (Bacillus thuringiensis). Four isolates were identified at genus level as Metschnikowia (1) and Saccharomyces (3). However 4 isolates could not be identified. In the fermented CCT with buckwheat flour, 31 of the total 38 isolates were identified. The species of the 14 yeasts were found as H. uvarum (5), T. delbrueckii (1), Wickerhamomyces anomalus (1), S. cerevisiae (2), C. krusei (3), C. lusitaniae (1), and C. kefyr (1). Furthermore, 15 yeast isolates were in the genera of Candida (5), Metschnikowia (6), Hanseniaspora (1), and Saccharomyces (3) and two bacteria isolates were identified as Bacillus spp.
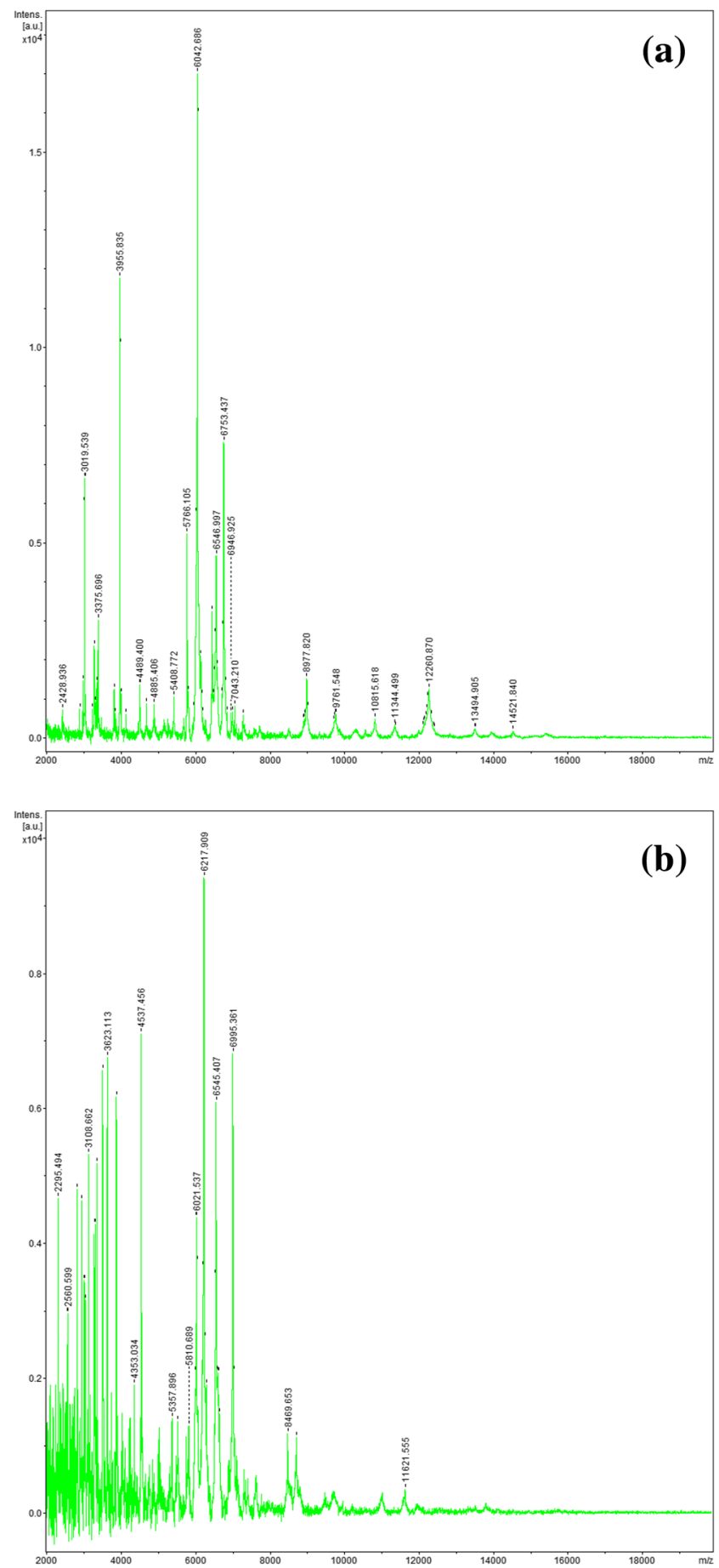

Fig. 1 MALDI-TOF/TOF mass spectra of strains H. uvarum MP-1 (a) and $S$. cerevisiae LP-6 (b)

When the identification results of the non-fermented CCT with clear flour were investigated, it could be revealed that 17 of the total 20 isolates were identified. Fourteen of the isolates were identified at species level as $H$. uvarum (4), S. cerevisiae (4), T. delbrueckii (4), C. lusitaniae (1), and Bacillus oleronius (1), in addition to genus identifications as Metschnikowia (1), Candida (1), and Bacillus (1). For the fermented CCT with clear flour, 21 of the 32 isolates were identified at species 
level, 17 of which were yeasts (H. uvarum, 7; T. delbrueckii, 3 , C. krusei, 3; W. anomalus, 1; Candida parapsilosis, 1 and C. lusitaniae, 2) and four of them were bacteria (Bacillus cereus, 2; Bacillus licheniformis, 2). The genus identifications were as Candida (4), Metschnikowia (3), 1 Wickerhamomyces (1), Saccharomyces (1), and Bacillus (2) and 12 of the total 44 isolates could not be identified (Table 1).

Identified (161) yeast isolates were in seven different genera and their percentage could be summarized as the following: Candida, 30.4\%; Hanseniaspora, 19.9\%; Saccharomyces, $19.3 \%$; Metschnikowia, 14.9\%; Pichia, 1.9\%; Wickerhamomyces, $1.9 \%$; and Cyberlindnera, $1.2 \%$. Ninetyseven isolates were identified at species level and belonged to ten different species which were $H$. uvarum (32\%), S. cerevisiae (19.6\%), T. delbrueckii (18.6\%), C. krusei (11.3\%), C. lusitaniae (9.3\%), M. pulcherrima (3.1\%), W. anomalus (2.1\%), C. kefyr (2.1\%), Cy. fabianii (1\%), and C. parapsilosis (1\%). Although the microflora of CCT samples was dominated by Candida genus $(30.4 \%)$, the mostly identified species was found to be H. uvarum.

Results of species identifications in accordance with different production stages were given in Figs. 2 and 3. Since the microflora of CCT samples was determined to be yeast dominated, only the identified yeast species were included in these figures. The identification results of the non-fermented and fermented CCT produced from wheat flour were shown in Fig. 2. S. cerevisiae was determined to be the main species in the dough of the non-fermented CCT with wheat flour, as well as H. uvarum, C. lusitaniae, C. krusei, S. cerevisiae, and T. delbrueckii. However, H. uvarum and T. delbrueckii along with $M$. pulcherrima could be isolated after the first drying of the non-fermented tarhana. For the fermented CCT with wheat flour, $C$. krusei was the only species to be isolated in dough. Furthermore, the fermentation process stimulated the growth of H. uvarum, M. pulcherrima, and Pichia spp. Although no yeast was identified after the final drying of the non-fermented product, T. delbrueckii was the only species isolated from the fermented product even after the final drying.

The predominant species was observed to be $S$. cerevisiae for non-fermented CCT produced with wholegrain hull-less barley flour as shown in Table 1. H. uvarum, C. lusitaniae, and $S$. cerevisiae species isolated from cornelian cherry puree as well as $C$. kefyr were also encountered in the tarhana dough (Fig. 2). However, only $C$. lusitaniae and $S$. cerevisiae as well as T. delbrueckii could be identified after the first drying of the non-fermented tarhana. Fermentation process stimulated the growth of M. pulcherrima and Pichia spp. The mostly isolated species $S$. cerevisiae in the non-fermented product did not appear until the first drying stage of the fermented tarhana. T. delbrueckii was isolated only from the final product of the fermented tarhana. T. delbrueckii was unexpectedly isolated from the dried and final products of CCT with wholegrain hull-less barley flour, although it could not be detected in the cornelian cherry and other stages of the production.

The results of species identification and isolation sources of the yeasts for CCT with buckwheat flour could be seen in Fig. 3 . $S$. cerevisiae was the main species found in the non-fermented tarhana similarly to the non-fermented CCT with wheat and wholegrain hull-less barley flour. However, the predominant species were H. uvarum and C. krusei for the fermented CCT. The species of $S$. cerevisiae, $H$. uvarum, and T. delbrueckii were isolated from the dough and first dried product of the nonfermented tarhana. In the fermented CCT with buckwheat flour, H. uvarum probably originated from cornelian cherry was isolated at all time points of fermentation besides tarhana dough. The growth of $C$. krusei had been promoted by fermentation and it was also isolated after the first drying of the fermented CCT. $W$. anomalus was the distinct species which was only detected in the 48th hours of the fermentation.

In the production of non-fermented CCT with clear flour, S. cerevisiae, H. uvarum, and T. delbrueckii were observed as predominant species. As could be seen from Fig. 3, the species H. uvarum, C. lusitaniae, and S. cerevisiae belonging to cornelian cherry were also isolated from non-fermented tarhana dough. However, only H. uvarum could be observed in dried tarhana. Species of T. delbrueckii was isolated from the dough and final product of the non-fermented tarhana. For the fermented tarhana, it was emphasized that H. uvarum, which was isolated from dough, survived during the fermentation process similarly to other fermented products. H. uvarum was detected also to be predominant species for the fermented CCT with clear flour. Besides other yeasts, W. anomalus was isolated at the 48th hours of the fermentation, similar to the fermented buckwheat flour tarhana.

In this study, in addition to majority of yeasts, a few of bacteria were also isolated in CCT products with buckwheat flour and clear flour. For buckwheat flour tarhana, these isolates were obtained from dough of the non-fermented and fermented buckwheat tarhana and they were identified as $B$. thuringiensis and Bacillus spp., respectively. One of the Bacillus spp. was also isolated from the final product of the fermented CCT with buckwheat flour. In addition, Bacillus oleronius and Bacillus spp. were originated from the dough and final product of the nonfermented tarhana, in addition to $B$. cereus, B. licheniformis, and Bacillus spp. which were isolated from production stages of the fermented CCT produced with clear flour.

The distances between the isolates from cornelian cherry puree and CCT with wheat flour were demonstrated by the dendogram in Fig. 4. It could be seen that $C$. lusitaniae LKH-4 was very close to puree isolates (LP7 and LP9) indicating that this strain isolated from tarhana dough was originated from cornelian cherry puree. $C$. krusei LP-2 strain of cornelian cherry puree was determined to be close to (0.2) C. krusei LKH-1 which was isolated from non-fermented tarhana dough. However, strains of C. krusei LFKH-1, LFKD-2, and LFKHc-1 obtained 
Fig. 2 Identified yeast species from different production steps of the CCT produced with wheat flour (a) and wholegrain hull-less barley flour (b) (F, fermented; NF, non-fermented) (a)

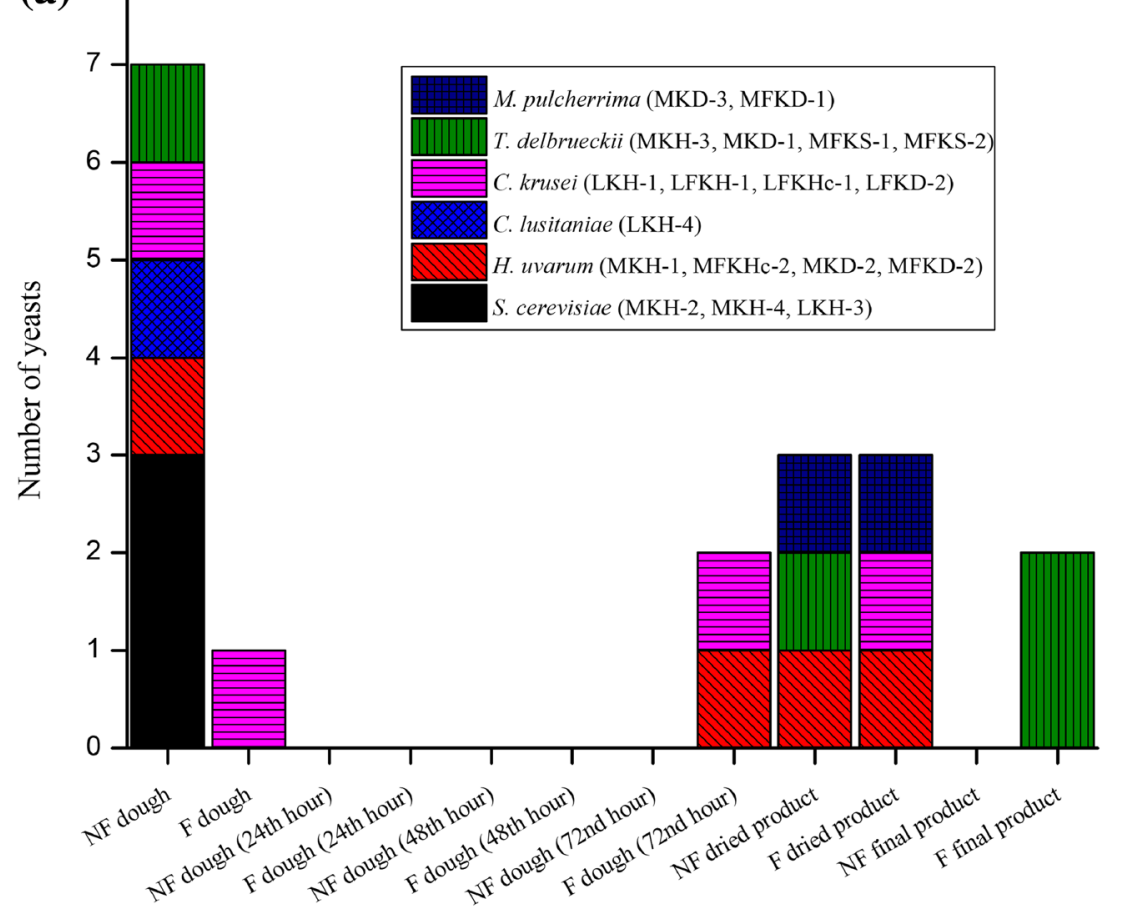

(b)

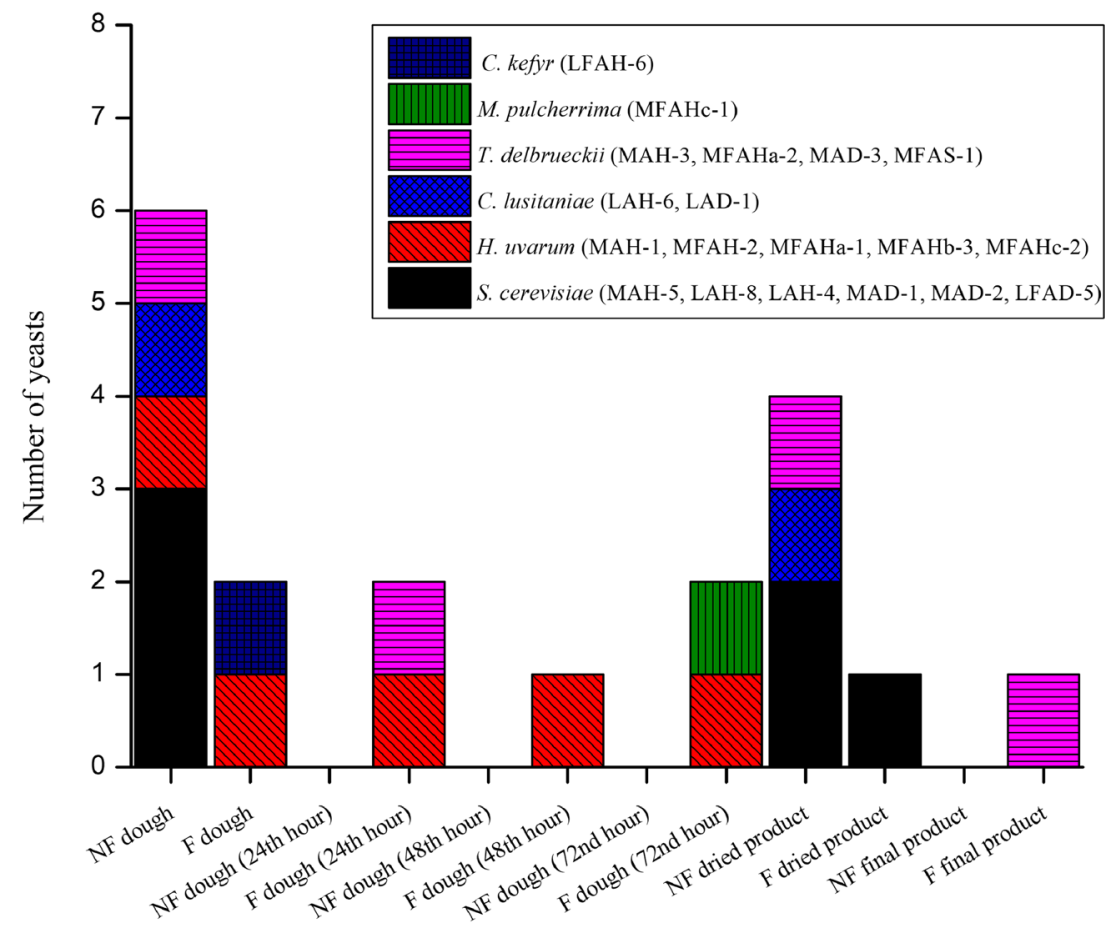

from the fermented tarhana clustered as a distinct branch and they were found to be close to each other. S. cerevisiae strains encountered in tarhana dough (MKH-2 and MKH-4) were relatively distinct from (0.5) S. cerevisiae LP-6. As it was shown, H. uvarum strains obtained from cornelian cherry puree were distinct from particularly the strain of MFKD-2 (0.7) indicating that its source is probably different from puree.
It was observed from Fig. 4 that $C$. lusitaniae LAH-6 and LAD-1 strains isolated from the non-fermented hull-less barley flour tarhana dough and dried tarhana were probably also encountered in cornelian cherry puree, as they were very close to LP-9 and LP-7 strains. All H. uvarum strains of cornelian cherry puree and the strain MAH-1 originating from the nonfermented tarhana dough clustered together with all fermented 
Fig. 3 Identified yeast species from different production steps of the CCT produced with buckwheat flour (a) and clear flour (b) (F, fermented; NF, nonfermented)

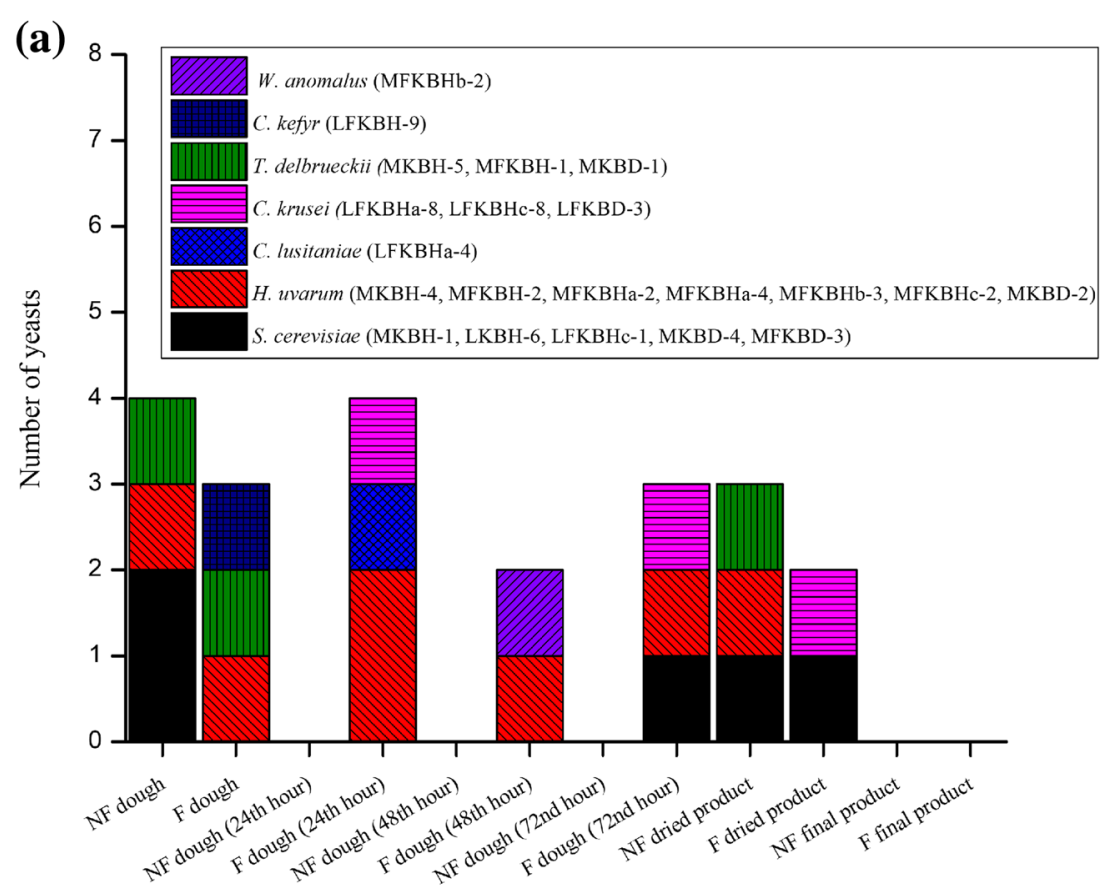

(b)

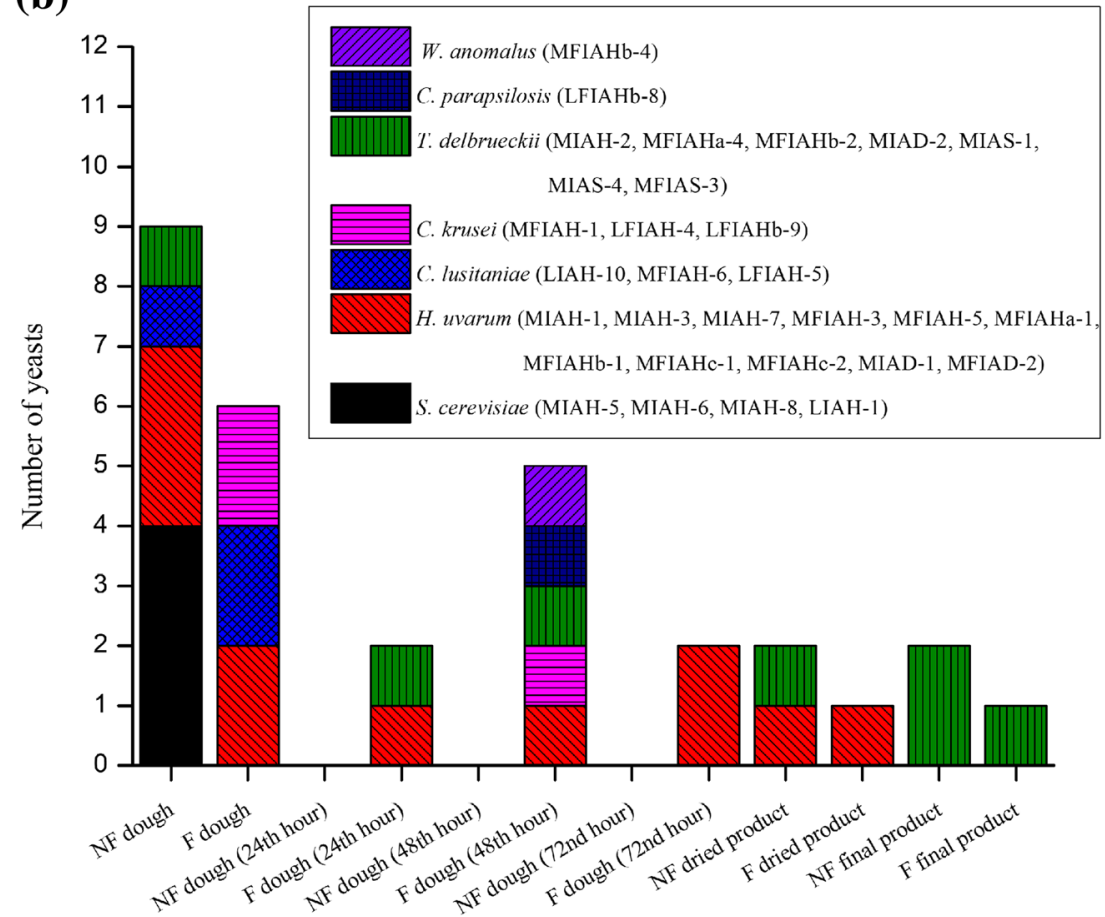

tarhana isolates at a distance level of 0.5 . The strains of MFAH-2 and MFAHa-1 isolated from the fermented tarhana dough and the 24th hour of dough fermentation, respectively, were very similar to each other. It could be seen from Fig. 2 that $S$. cerevisiae LAH-4, MAD-1, and LFAD-5 were close to each other. S. cerevisiae LP-6 and MAH-5 clustered together and they were distinct from other $S$. cerevisiae strains.

As shown in Fig. 5, C. krusei LP-2 did not cluster together with strains of $C$. krusei LFKBD-3 and LFKBHa- 8 in the dendogram demonstrating strains of cornelian cherry puree and CCT with buckwheat flour. Although C. lusitaniae LP-7 and LP-9 strains obtained from puree were close to each other, C. lusitaniae LFKBHa-4 was relatively distinct from (0.4) these isolates. All $S$. cerevisiae strains in CCT with buckwheat flour clustered together. The strains of MKBD-4 and MKBH1 were very close to each other. $H$. uvarum strains of cornelian cherry puree clustered as a distinct branch and they were relatively distinct from other $H$. uvarum strains. 
Fig. 4 Cluster analysis of MALDI-TOF/TOF mass spectra of strains belonging to CCT productions performed with wheat flour (a) and wholegrain hull-less barley flour (b) and their comparison to strains of cornelian cherry puree $(\mathrm{F}$, fermented; $\mathrm{P}$, cornelian cherry puree; $\mathrm{K}$, CCT produced with wheat flour; A, CCT produced with hull-less barley flour; $\mathrm{H}$, dough; a, b and c, 24th, 48th, and 72nd hours of fermentation, respectively; D, dried product; $S$, final product; $M$ and $\mathrm{L}$, specific codes independent from process stages)

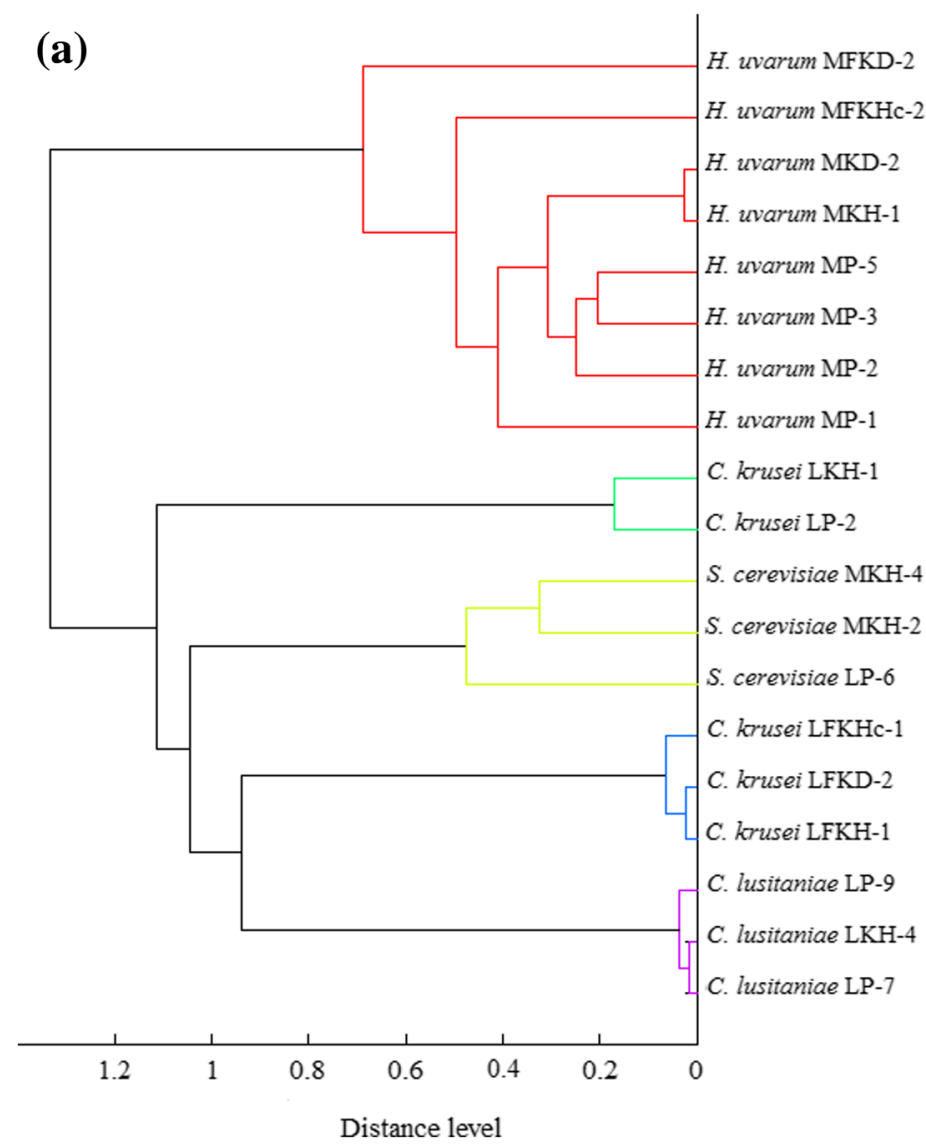

(b)

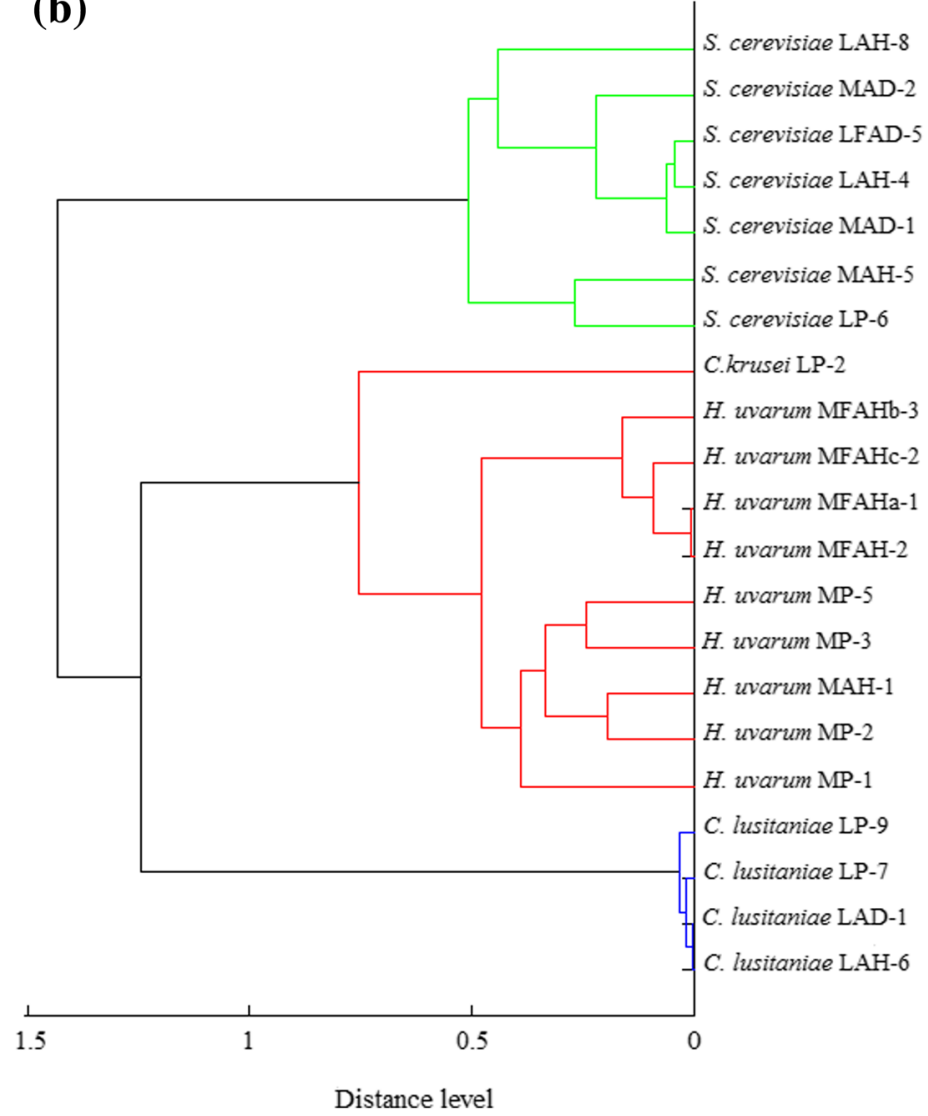


Fig. 5 Cluster analysis of MALDI-TOF/TOF mass spectra of strains belonging to CCT productions performed with buckwheat flour (a) and clear flour (b) and their comparison to strains of cornelian cherry puree (F, fermented; $\mathrm{P}$, cornelian cherry puree; KB, CCT produced with buckwheat flour; IA, CCT produced with clear flour; $\mathrm{H}$, dough; a, b, and c, 24th, 48th, and 72nd hours of fermentation, respectively; $\mathrm{D}$, dried product; $\mathrm{S}$, final product; $\mathrm{M}$ and $\mathrm{L}$, specific codes independent from process stages)

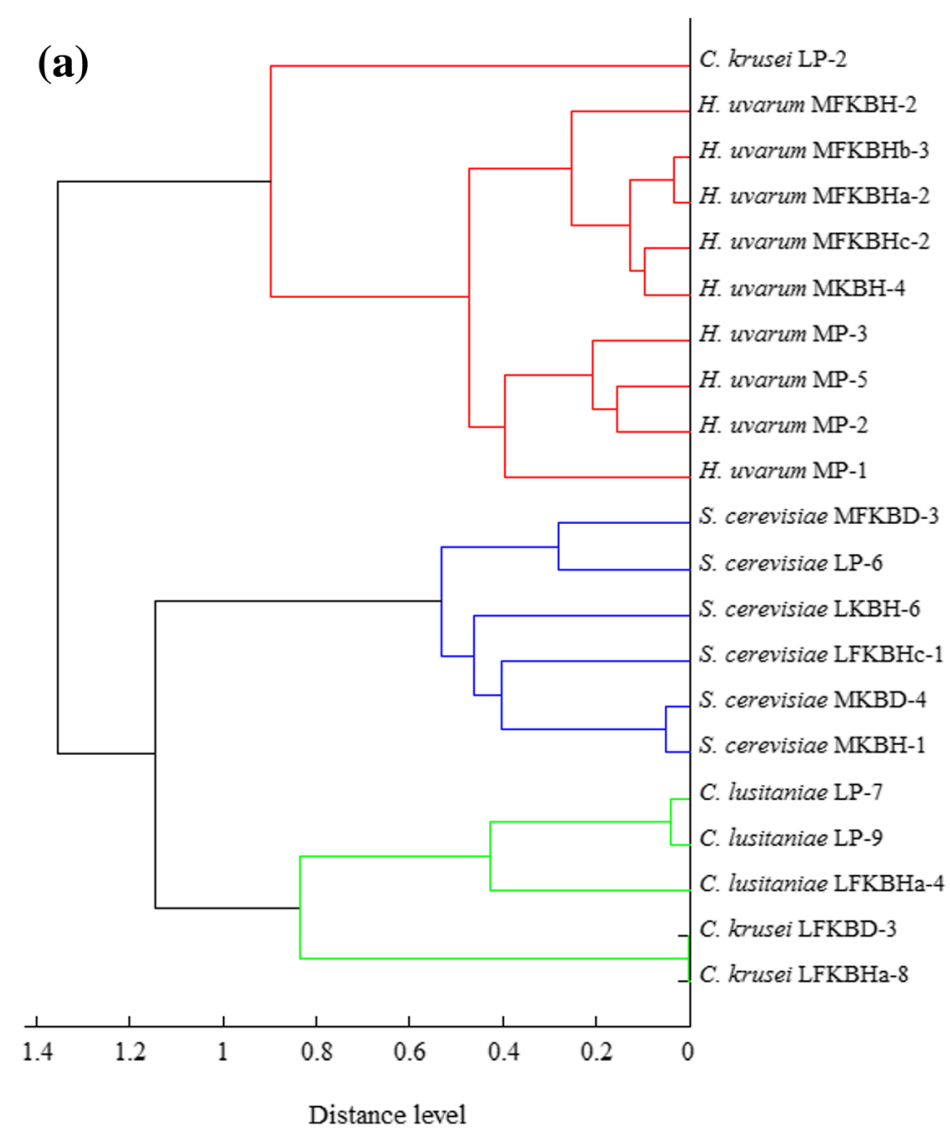

(b) S. cerevisiae MIAH-5 S. cerevisiae LIAH-1 S. cerevisiae MIAH-6 S. cerevisiae MIAH-8

S. cerevisiae LP-6

C. lusitaniae LIAH-10

C. lusitaniae LP-7

C. lusitaniae MFIAH-6

C. lusitaniae LFIAH-5

C. lusitaniae LP-9

H. uvarum MFIAD-2

H. uvarum MFIAHc-2

H. uvarum MFIAHa-1

H. uvarum MIAH-7

H. uvarum MFIAHc-1

H. uvarum MFIAH-3

H. uvarum MIAH-1

H. uvarum MP-5

H. uvarum MP-3

H. uvarum MP-2

H. uvarum MFIAH-5

H. uvarum MIAD-1

H. uvarum MIAH-3

H. uvarum MP-1

C. krusei LFIAH-4

H. uvarum MFIAHb-1

C. krusei LFIAHb-9

C. krusei MFIAH-1

C. krusei LP-2

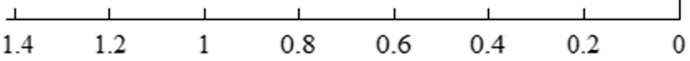

Distance level 
It was demonstrated in Fig. 5 that $C$. krusei strains isolated from cornelian cherry puree (LP-2) and fermented CCT with clear flour (MFIAH-1) were determined to be similar strains. C. lusitaniae LP-9 and LFIAH-5 were also very close to each other. It could be observed that some $H$. uvarum strains such as MFIAH-3 and MIAH-1 were close to each other; however, they clustered as a distinct branch from cornelian cherry puree isolates. Strains of S. cerevisiae obtained from tarhana dough, particularly $S$. cerevisiae MIAH-5, might be distinct from S. cerevisiae LP-6 which was encountered in puree, and could be originated from process equipment.

\section{Discussion}

In this study, CCT productions were performed by traditional method and also by fermentation. Microflora of CCT products was investigated for the first time during different production stages. The species of $H$. uvarum (anamorph: Kloeckera apiculata), which have been typically isolated from several production stages of CCT products, is one of the apiculate yeasts involved in the spontaneous fermentation of grape fruit together with S. cerevisiae (Tristezza et al. 2016). It could be emphasized that yeast microflora of the tarhana products was clearly dominated by the $H$. uvarum. Besides, the only yeast species isolated from the final products of some non-fermented CCT samples and also from majority of fermented CCT products was observed as T. delbrueckii. Species of T. delbrueckii occur in fruit juices and spontaneously fermented food because of its specific properties including the use of maltose and lactate, producing ester compounds and different fruity aromas in addition to being osmotolerant to $10 \% \mathrm{NaCl}$ or $5 \%$ glucose (De Vuyst et al. 2016; Canonico et al. 2017). So, these could be associated with the isolation of this species from cornelian cherry tarhana products containing approximately $5.5-6 \%$ of $\mathrm{NaCl}$. As a remarkable point, the species of $M$. pulcherrima known as one of the yeasts mainly isolated from fruits could not be isolated from cornelian cherry although it was detected in doughs and dried products of some CCT. It was noteworthy that Cy. fabianii was not isolated from any production stages of non-fermented or fermented CCT with clear flour, although it was detected in the clear flour. $C y$. fabianii (previously Hansenula fabianii, Pichia fabianii, and Lindnera fabianii) is a yeast species known as a human pathogen (Joo et al. 2015). In addition to the yeasts, Bacillus spp. (11) were identified from CCT products. It was reported that Bacillus spp., which are Gram-positive spore forming bacteria, were isolated from biofilms in several environments such as food and beverage industry, and endospores of these bacteria could also contaminate raw materials such as flour (Viedma et al. 2011; Faille et al. 2014).

A limited number of studies were conducted to identify lactic acid bacteria (Sengun et al. 2009; Settanni et al. 2011; Simsek et al. 2017) and yeast microflora (Settanni et al. 2011; Ozel et al.
2015) of traditional tarhana products. In a reported study, several lactic acid bacteria isolates including 12 species from 6 different genera as Pediococcus, Streptococcus, Lactobacillus, Enterococcus, Leuconostoc, and Weissella were identified using a combination of pheno and genotypic methods in tarhana products collected from eight different cities of Turkey (Sengun et al. 2009). In the other study, lactic acid bacteria isolates from tarhana dough fermentation were identified to be Pediococcus acidilactici, Lactobacillus plantarum, and Lactobacillus brevis by a combined genetic approach consisting of 16S/23S rRNA intergenic spacer region (ITS) and partial 16S rRNA gene sequencing (Settanni et al. 2011). The main species for homemade tarhana was found to be L. plantarum by Simsek et al. (2017) and it was also reported that microflora of commercial tarhana dough fermentation predominantly composed of $L$. brevis and Lactobacillus alimentarius. In the present study, LAB could not be isolated from CCT products and the microflora was mainly composed of yeasts. The major reason of this could be that CCT differed from traditional tarhana and yoghurt is not used in the formulation of CCT products while cornelian cherry is the main ingredient.

In a reported study, the yeast isolates of homemade and commercial traditional tarhana dough were identified to be species of Pichia kudriavzevii, Candida glabrata, Candida humilis, Saccharomyces cerevisiae, Kluyveromyces marxianus, Kazachstania servazzi, and Kazachstania unispora by using analysis of LSU and ITS-5.8S rDNA sequence (Ozel et al. 2015). In another study, the yeasts from tarhana fermentation were identified by the restriction fragment length polymorphism (RFLP) of the 5.8S ITS rRNA gene to be Rhodotorula glutinis and also predominantly S. cerevisiae (Settanni et al. 2011). As could be seen from those studies, the yeast microflora of traditional tarhana was reported to be rich in S. cerevisiae similarly to our study. However, CCT is a special product that differs from classical tarhana, being mainly composed of cornelian cherry instead of yoghurt. Therefore, its yeast microflora also included H. uvarum, M. pulcherrima, T. delbrueckii, W. anomalus, and Candida spp. The distinctness in formulation of CCT products might be possible reason to its unique microflora and diverse yeast species.

It is obvious from the results that the fermentation process promoted both yeast growth and diversity of yeast genus and species. Although common species were found in both nonfermented and fermented products, Wickerhamomyces spp. and Pichia spp. along with C. parapsilosis, W. anomalus, and C. kefyr could be isolated from only fermented CCT.

MALDI-TOF mass spectrometry is a novel high throughput, fast, and cost-effective identification method based on the analysis of whole cell proteins, which offers the possibility of accurately identify to the genus, species, and subspecies levels belong to clinical, enviromental, and foodborne bacteria and yeast isolates (Lv et al. 2016; Quintilla et al. 2018). A MALDI-TOF mass spectrometer is composed of an ion source for solid samples 
coupled to mass analyzer that sorts the ions according to their time-of-flight to travel a given distance in a free-field environment (Nacef et al. 2017). There are only a few studies on identifying the microorganisms and particularly yeasts from traditional fermented food products by using MALDI-TOF MS. Some of these studies were about evaluation of lactic acid bacteria and yeast identification results obtained from both proteomic approach using MALDI-TOF MS and genotypic analyzing methods. In a study conducted by Kim et al. (2017), rapid identifying capability of MALDI-TOF MS was confirmed that Weisella species isolated from Korean fermented foods and previously identified by $16 \mathrm{~S}$ rRNA sequencing could be also correctly re-identified. In another study, the same lactic acid bacteria species of traditional French cheese could be identified from both of the analyzing methods, even though two of the 10 isolates were practically identified at genus level by MALDI-TOF MS (Nacef et al. 2017). To identify the bacterial communities of traditional fermentation starters for Chinese rice wine, the MALDI-TOF MS was demonstrated to be a faster and more accurate method because of particularly being useful for the discrimination of closely related species such as Bacillus spp. and L. plantarum group (Lv et al. 2016). It was reported by Spitaels et al. (2015) that the yeast isolates obtained from traditional beer fermentation were identified through both MALDI-TOF MS and D1/D2 26S rRNA or ACT1 gene sequences analysis. On the other hand, there have been also some studies using only genotypic methods to identify microorganisms of several traditional cereal-based fermented foods and beverages (Osorio-Cadavid et al. 2008; Kumar et al. 2010; Sekwati-Monang and Gänzle 2011; Pedersen et al. 2012).

Apart from the reported studies, MALDI-TOF/TOF MS which provides double resolution was used in the present study instead of MALDI-TOF MS (Suckau et al. 2003). Approximately $75 \%$ of the isolates could be identified and the microflora of CCT products was investigated for the first time. Dendograms showed that some of the yeast species isolated during production stages were originated from cornelian cherry although source of the others were probably process equipment. The yeast microflora of CCT samples including several species of Candida, Hanseniaspora, Metschnikowia, Pichia, Saccharomyces, and Torulaspora was determined to be substantially similar to microflora of wine. As it was reported by Capozzi et al. (2015), these yeast species were encountered also in grape and wine environments. The possible reason of this similarity could be the high amount of cornelian cherry fruit in the CCT formulation.

\section{Conclusion}

With this study, microflora of cornelian cherry tarhana produced from different raw materials (bread wheat, wholegrain hull-less barley, buckwheat, and clear flours) and methods were demonstrated for the first time. Since cornelian cherry was found to be rich in yeast count, microflora during CCT production was also dominated by yeasts. Common yeast species isolated from all CCT productions were determined to be $H$. uvarum, S. cerevisiae, T. delbrueckii, M. pulcherrima, C. krusei, and C. lusitaniae. In addition, a few number of lactic acid bacteria could be detected in only raw materials and 11 isolates of Bacillus spp. were obtained from production stages of fermented CCT with buckwheat and clear flours. Fermentation induced both yeast growth and diversity. The use of dendograms gave idea about the origins of the strains. This study introduced novel species-identified, endogenic yeasts which could have potential technological characteristics.

Acknowledgements The authors would like to thank the Scientific, Industrial and Technological Application and Research Center of Bolu Abant Izzet Baysal University for identification of the isolates by MALDI-TOF/TOF MS.

Funding This research was financially supported by Scientific Research Projects coordination Unit of Bolu Abant Izzet Baysal University, Bolu, Turkey (project number: BAP- 2016.09.04.1084).

\section{Compliance with ethical standards}

Conflicts of interest The authors declare that they have no conflict of interest.

Research involving human participants and/or animals N/A

Informed consent N/A

Publisher's note Springer Nature remains neutral with regard to jurisdictional claims in published maps and institutional affiliations.

\section{References}

Cagindi O, Aksoylu Z, Savlak NY, Kose E (2016) Comparison of physicochemical and functional properties of domestic and commercial tarhana in Turkey. Bulg J Agric Sci 22(2):324-330

Canonico L, Comitini F, Ciani M (2017) Torulaspora delbrueckii contribution in mixed brewing fermentations with different Saccharomyces cerevisiae strains. Int J Food Microbiol 259:7-13

Capozzi V, Garofalo C, Chiriatti MA, Grieco F, Spano G (2015) Microbial terroir and food innovation: the case of yeast biodiversity in wine. Microbiol Res 181:75-83

De Vuyst L, Harth H, Van Kerrebroeck S, Leroy F (2016) Yeast diversity of sourdoughs and associated metabolic properties and functionalities. Int J Food Microbiol 239:26-34

Dinda B, Kyriakopoulos AM, Dinda S, Zoumpourlis V, Thomaidis NS, Velegraki A, Markopoulos C, Dinda M (2016) Cornus mas L. (cornelian cherry), an important European and Asian traditional food and medicine: Ethnomedicine, phytochemistry and pharmacology for its commercial utilization in drug industry. J Ethnopharmacol 193:670-690

Faille C, Bénézech T, Midelet-Bourdin G, Lequette Y, Clarisse M, Ronse G, Ronse A, Slomianny (2014) Sporulation of Bacillus spp. within 
biofilms: a potential source of contamination in food processing environments. Food Microbiol 40:64-74

Joo EJ, Yeom JS, Kwon MJ (2015) Successful treatment of Cyberlindnera fabianii with anidulafunginin a case of catheterrelated infection. 10th International Symposium on Antimicrobial Agents and Resistance, Korea, May 14-15

Kabak B, Dobson ADW (2011) An introduction to the traditional fermented foods and beverages of Turkey. Crit Rev Food Sci Nutr 51(3):248-260

Kim E, Cho Y, Lee Y, Han SK, Kim CG, Choo DW, Kim YR, Kim HY (2017) A proteomic approach for rapid identification of Weissella species isolated from Korean fermented foods on MALDI-TOF MS supplemented with an in-house database. Int J Food Microbiol 243: 9-15

Kumar RS, Varman DR, Kanmani P, Yuvaraj N, Paari KA, Pattukumar V, Arul V (2010) Isolation, characterization and identification of a potential probiont from South Indian fermented foods (Kallappam, Koozh and Mor Kuzhambu) and its use as biopreservative. Probiotics Antimicro 2(3):145-151

Lv XC, Jia RB, Li Y, Chen F, Chen ZC, Liu B, Chen SJ, Rao PF, Ni L (2016) Characterization of the dominant bacterial communities of traditional fermentation starters for Hong Qu glutinous rice wine by means of MALDI-TOF mass spectrometry fingerprinting, 16S rRNA gene sequencing and species-specific PCRs. Food Control 67:292-302

Marco ML, Heeney D, Binda S, Cifelli CJ, Cotter PD, Foligné B, Gänzle M, Kort R, Pasin G, Pihlanto A, Smid EJ, Hutkins R (2017) Health benefits of fermented foods: microbiota and beyond. Curr Opin Biotechnol 44:94-102

Nacef M, Chevalier M, Chollet S, Drider D, Flahaut C (2017) MALDITOF mass spectrometry for the identification of lactic acid bacteria isolated from a French cheese: the Maroilles. Int J Food Microbiol 247:2-8

Osorio-Cadavid E, Chaves-López C, Tofalo R, Paparella A, Suzzi G (2008) Detection and identification of wild yeasts in Champús, a fermented Colombian maize beverage. Food Microbiol 25(6):771777

Ozdemir S, Gocmen D, Yildirim Kumral A (2007) A traditional Turkish fermented cereal food: Tarhana. Food Rev Int 23(2):107-121

Ozel S, Sabanoglu S, Con AH, Simsek O (2015) Diversity and stability of yeast species during the fermentation of tarhana. Food Biotechnol 29(1):117-129

Pedersen LL, Owusu-Kwarteng J, Thorsen L, Jespersen L (2012) Biodiversity and probiotic potential of yeasts isolated from Fura, a West African spontaneously fermented cereal. Int J Food Microbiol 159(2):144-151
Quintilla R, Kolecka A, Casaregola S, Daniel HM, Houbraken J, Kostrzewa M, Boekhout T, Groenewald M (2018) MALDI-TOF MS as a tool to identify foodborne yeasts and yeast-like fungi. Int J Food Microbiol 266:109-118

Schulthess B, Brodner K, Bloemberg GV, Zbinden R, Böttger EC, Hombach M (2013) Identification of Gram-positive cocci by use of matrix-assisted laser desorption ionization -time of flight mass spectrometry: comparison of different preparation methods and implementation of a practical algorithm for routine diagnostics. J Clin Microbiol 51(6): 1834-1840

Sekwati-Monang B, Gänzle MG (2011) Microbiological and chemical characterisation of ting, a sorghum-based sourdough product from Botswana. Int J Food Microbiol 150(2-3):115-121

Sengun IY, Nielsen DS, Karapinar M, Jakobsen M (2009) Identification of lactic acid bacteria isolated from Tarhana, a traditional Turkish fermented food. Int J Food Microbiol 135(2):105-111

Settanni L, Tanguler H, Moschetti G, Reale S, Gargano V, Erten H (2011) Evolution of fermenting microbiota in tarhana produced under controlled technological conditions. Food Microbiol 28(7):1367-1373

Simsek O, Ozel S, Con AH (2017) Comparison of lactic acid bacteria diversity during the fermentation of Tarhana produced at home and on a commercial scale. Food Sci Biotechnol 26(1):181-187

Spitaels F, Wieme AD, Janssens M, Aerts M, Van Landschoot A, Vuyst LD, Vandamme P (2015) The microbial diversity of an industrially produced lambic beer shares members of a traditionally produced one and reveals a core microbiota for lambic beer fermentation. Food Microbiol 49:23-32

Suckau D, Resemann A, Schuerenberg M, Hufnagel P, Franzen J, Holle A (2003) A novel MALDI LIFT-TOF/TOF mass spectrometer for proteomics. Anal Bioanal Chem 376(7):952-965

Tanguler H (2014) Traditional Turkish fermented cereal based products: Tarhana, boza and chickpea bread. Turkish JAF Sci Tech 2(3):144 149

Tristezza M, Tufariello M, Capozzi V, Spano G, Mita G, Grieco F (2016) The oenological potential of Hanseniaspora uvarum in simultaneous and sequential co-fermentation with Saccharomyces cerevisiae for industrial wine production. Front Microbiol 7:670

Viedma PM, Abriouel H, Omar NB, López RL, Gálvez A (2011) Inhibition of spoilage and toxigenic Bacillus species in dough from wheat flour by the cyclic peptide enterocin AS-48. Food Control 22: 756-761 